[6] G. J. Rieger, Elementare Lösung des Waringschen Problems für algebraische Zahlkörper mit der verallgemeinerten Linnikschen Methode, Math. Annalen 148 (1962), pp. $83-88$.

[7] C. L. Siegel, Generalisation of Waring's problem to algebraic number fields, Amer. J. Math. 66 (1944), pp. $122-136$.

[8] - Sums of m-th powers of algebraic integers, Ann. Math. 46 (1945), pp. 313 . 339.

[9] R. M. Stemmler, The easier Waring problem in algebraic number fields, Acta Arithmetica 6 (1961), pp. 447-468.

[10] A. Weil, Numbers of solutions of equations in finite fields, Bull. Amer. Math. Soc. 55 (1949), pp. 497-508.

[11] C. P. Ramanujam, Sums of $m$-th powers in p-adic rings, Mathematika 10 (1963), pp. 137-146.

UNIVERSITY OF MANOHESTER

Reģu par la Rédaction le 8. 10. 1963

\section{Tauberian theorems for sum sets}

by

P. ERdös (London), B. GordoN* (Los Angeles, Calif.),

L. A. Rubel (Urbana, Ill.) and E. G. Straus (Los Angeles, Calif.)

Introduction. The sums formed from the set of non-negative powers of 2 are just the non-negative integers. It is easy to obtain "abelian" results to the effect that if a set is distributed like the powers of 2 , then the sum set will be distributed like the non-negative integers. We will be concerned here with converse', or "Tauberian" results. The main theme of this paper is the following question: if the set of sums formed from a given set of positive real numbers resembles an arithmetic progression, how much must the original set resemble a set of constant multiples of powers of 2 ?

If we denote the given set by $k_{0}, k_{1}, k_{2}, \ldots$, arranged in ascending order, and let $S(x)$ count the number of those sums of distinct $k_{j}$ that do not exceed $x$, our problem is, roughly, that of showing that $k_{n}$ is close to $2^{n}$ if $S(x)$ is close to $x$. Our first result gives sharp bounds for liminf and limsup of $2^{n} / k_{n}$ in terms of liminf and limsup of $S(x) / x$. In the next section, we show that if $S(x)-x$ is bounded, then $k_{n}-2^{n}$ is bounded, and furthermore, $\sum\left|k_{n}-2^{n}\right|<\infty$, so that if the $k_{n}$ are integers, then $k_{n}=2^{n}$ for all large $n$. We extend the method in the succeeding section to obtain estimates for $k_{n}-2^{n}$ and $\sum_{n \leqslant N}\left|k_{n}-2^{n}\right|$ in terms of suitable bounds for $S(x)-x$, even if $S(x)-x$ is unbounded. Finally, on a slightly different note, we show that it is not possible for $S(x)$ to behave too much like $x^{a}$ if $\alpha<1$.

1. Asymptotic behavior. Let $K=k_{0}, k_{1}, k_{2}, \ldots, 0<k_{0} \leqslant k_{1} \leqslant k_{2}$ $\leqslant \ldots$, be any sequence of positive real numbers. Let $S(x)$ denote the number of choices of $\varepsilon_{0}, \varepsilon_{1}, \varepsilon_{2}, \ldots$ such that for each $j=0,1,2, \ldots$, either $\varepsilon_{j}=0$ or $\varepsilon_{j}=1$, and such that $\varepsilon_{0} k_{0}+\varepsilon_{1} \dot{k}_{1}+\ldots \leqslant x$. Let

$$
\begin{aligned}
A=\liminf _{x \rightarrow \infty} S(x) / x, & \alpha=\liminf _{n \rightarrow \infty} 2^{n} / k_{n}, \\
B=\limsup _{x \rightarrow \infty} S(x) / x, & \beta=\limsup _{n \rightarrow \infty} 2^{n} / k_{n} .
\end{aligned}
$$

* Alfred P. Sloan Fellow. 
A simple estimate shows that

$$
\alpha \leqslant A \text { and } \beta \geqslant B .
$$

We now give sharp inequalities in the opposite direction.

THEOREM 1. $\alpha=A$ and

$$
B \geqslant \beta\left(2 \frac{\alpha}{\beta}-\frac{a^{2}}{\beta^{2}}\right) .
$$

Inequality (2) is best possible in the sense that, given any $\alpha$ and $\beta$ with $\frac{1}{2}<$ $<\alpha \mid \beta \leqslant 1$, there exists a sequence $K$ for which equality holds, and given any $A$ and $B$ with $\frac{2}{3}<A / B \leqslant 1$, there exists a sequence $K$ for which equatity holds.

Remarks. It follows immediately from the theorem that

$$
\text { if } \lim _{x \rightarrow \infty} S(x) / x=\theta \neq 0, \text { then } \lim _{n \rightarrow \infty} 2^{n} / k_{n}=\theta .
$$

This result was proved by a different method in [1]. The question was raised in [1] whether the statement remains true for $\theta=0$. The answer is no, as the following example shows. Let $k_{2^{n}}=2^{2^{n}}$, and let $k_{2^{n}-r}=k_{2^{n}}$ for $0 \leqslant r<2^{n-1}$. It is easy to see that $S(x)=o(x)$. On the other hand, $2^{n} / k_{n}=1$ for infinitely many $n$. It is easy to modify the example so that the $k_{n}$ are distinct, but it seems difficult to satisfy the additional condition, described in [1], that the sums of the $k_{n}$ are all distinct.

It seems likely that our methods, if carried out in greater detail, would yield an estimate similar to (2), but taking account of the integral part of $\log _{2} \beta / \alpha$, and that such an estimate would be best possible for any range of $\alpha / \beta$, and not merely for $\alpha / \beta>\frac{1}{2}$. Finally, if we permit $\varepsilon_{j}=0,1, \ldots, N-1$, then it seems likely that our methods will yield analogous results for the limsup and liminf of $N^{n} / k_{n}$.

Proof of the estimates. First, $S\left(k_{n}-1\right) \leqslant 2^{n}$, since if $\varepsilon_{0} k_{0}+$ $+\varepsilon_{1} k_{1}+\ldots+\varepsilon_{n} k_{n}+\ldots \leqslant k_{n}-1$, then $\varepsilon_{n}=\varepsilon_{n+1}=\ldots=0$, so that there are at most $2^{n}$ suitable choices of $\left\{\varepsilon_{j}\right\}$. Hence

$$
\frac{S\left(k_{n}-1\right)}{k_{n}-1} \leqslant \frac{2^{n}}{k_{n}-1}=\frac{2^{n}}{k_{n}} \cdot \frac{k_{n}}{k_{n}-1}
$$

and on letting $n \rightarrow \infty$, we get $A \leqslant \alpha$.

To obtain the estimate (2), we may suppose that $\alpha>0$, since if $a=0$ then (2) is trivially true. We now choose any $a>1 / \alpha$, so that $k_{n} \leqslant 2^{n} a$ for all sufficiently large $n$. Without loss of generality, we shall suppose that $k_{n} \leqslant 2^{n} a$ for all $n=0,1,2, \ldots$, because for any two sequences $K$ and $K^{\prime}$ with $k_{n}=k_{n}^{\prime}$ for $n \geqslant n_{0}$, it is easy to show that $A=A^{\prime}, B=$
$=B^{\prime}, \alpha=\alpha^{\prime}, \beta=\beta^{\prime}$. And given any $b$ with $b>1 / \beta$ we will have $k_{n} \leqslant 2^{n} b$ for infinitely many $n$.

We now choose $n$ large, with $k_{n} \leqslant 2^{n} b$, and estimate $S\left(2^{n} a\right)$. Clearly, $S\left(2^{n} a\right) \geqslant N_{1}+N_{2}$, where $N_{1}$ is the number of choices of $\left\{\varepsilon_{j}\right\}$, $j=0,1, \ldots, n-1$, such that

$$
\varepsilon_{0} k_{0}+\ldots+\varepsilon_{n-1} k_{n-1} \leqslant 2^{n} a
$$

and $N_{2}$ is the number of choices of $\left\{\varepsilon_{i}\right\}, j=0,1, \ldots, n-1$, such that

$$
\varepsilon_{0} k_{0}+\ldots+\varepsilon_{n-1} k_{n-1}+k_{n} \leqslant 2^{n} a .
$$

But if $\varepsilon_{0} 2^{0} a+\ldots+\varepsilon_{n-1} 2^{n-1} a \leqslant 2^{n} a$, then (3) holds, and therefore $N_{1} \geqslant 2^{n}$. And if $\varepsilon_{0} 2^{0} a+\ldots+\varepsilon_{n-1} 2^{n-1} a \leqslant 2^{n}(a-b)$, then (4) holds, so that

$$
N_{2} \geqslant\left[2^{n}\left(1-\frac{b}{a}\right)\right] \geqslant 2^{n}\left(1-\frac{b}{a}\right)-1 \text {. }
$$

Hence

$$
S\left(2^{n} a\right) \geqslant 2^{n}+2^{n}(1-b / a)-1, \quad \frac{S\left(2^{n} a\right)}{2^{n} a} \geqslant \frac{1}{a}\left(2-\frac{b}{a}\right)-\frac{1}{2^{n} a} .
$$

On letting $n \rightarrow \infty$ through a suitable sequence, we get

$$
B \geqslant \frac{1}{a}\left(2-\frac{b}{a}\right)=\frac{1}{b} \cdot \frac{b}{a}\left(2-\frac{b}{a}\right) .
$$

We may now let $a \rightarrow 1 / \alpha$ and $b \rightarrow 1 / \beta$ to obtain (2).

The estimate is best possible. To show that (2) is best possible, we prove the first part, that given any $\alpha$ and $\beta$ with $\frac{1}{2}<\alpha / \beta<1$, there exists. a $K$ such that $B=\beta\left(2 \alpha / \beta-\alpha^{2} / \beta^{2}\right)$. The second part then follows since $\varphi(\beta)=\beta\left(2 A \mid \beta-A^{2} / \beta^{2}\right)$ is a continuous function of $\beta$, with $\varphi(A)=A$ and $\varphi(2 A)=3 A / 2$, so that if we are given $A$ and $B$ with $1 \leqslant B / A<3 / 2$, we may apply the first part with $\alpha=A$ and $\beta$ such that $\varphi(\beta)=B$. For the construction of $K$, let $n_{m}$ be a sequence of positive integers that increases very rapidly to $\infty$. Let $a=1 / \alpha$ and $b=1 / \beta$ and define $k_{n}$ by $k_{n}=2^{n} a$ unless $n=n_{m}$ for some $m$, and $k_{n}=2^{n} b$ if $n=n_{m}$ for some $m$. The point of the restriction $a / \beta>\frac{1}{2}$ now appears; for the sequence $K$ to be suitably defined, we need $2^{n} b \geqslant 2^{n-1} a$, or $b / a \geqslant \frac{1}{2}$.

A simple argument now shows that $B \geqslant \limsup B_{n}$, where $B_{n}$ is defined as follows. Let $K^{n}$ be the sequence $\left\{k_{j}\right\}, j=0,1,2, \ldots$, where $k_{j}=2^{j} a$ for $j \neq n$ and $k_{j}=2^{j} b$ if $j=n$. Let $S_{n}(x)=S\left(x: K^{n}\right)$ and let $B_{n}=\sup \left(S_{n}(x) / x\right)$, where the supremum is over all values of $x \geqslant x_{0}(n)$, where $x_{0}(n)$ is a function of $n$ that tends very slowly to $+\infty$ as $n$ tends to $+\infty$.

To determine $S_{n}(x)$, we must count those $\left\{\varepsilon_{j}\right\}$ for which

$$
\varepsilon_{0} 2^{0} a+\ldots+\varepsilon_{n-1} 2^{n-1} a+\varepsilon_{n} 2^{n} b+\varepsilon_{n+1} 2^{n+1} a+\ldots \leqslant x .
$$


We now define $f_{n}(t)$ as the number of choices of $\left\{\varepsilon_{j}\right\}$ for which

$$
\varepsilon_{0} 2^{0}+\varepsilon_{1} 2^{1}+\ldots+\varepsilon_{n-1} 2^{n-1}+\varepsilon_{n+1} 2^{n+1}+\ldots \leqslant t .
$$

Now, considering in (5) the two cases $\varepsilon_{n}=0$ and $\varepsilon_{n}=1$, we see that

$$
S_{n}(x)=f_{n}\left(\frac{x}{a}\right)+f_{n}\left(\frac{x-2^{n} b}{a}\right) .
$$

If we write $y=x / a$, then from (7) we get

$$
\frac{S_{n}(a y)}{a y}=\frac{1}{a}\left\{\frac{f_{n}(y)}{y}+\frac{f_{n}\left(y-2^{n} b / a\right.}{y}\right\},
$$

so that

$$
B_{n}=\frac{1}{a} \sup \left\{\frac{f_{n}(y)+f_{n}\left(y-2^{n} b / a\right)}{y}\right\},
$$

where the supremum is over the range $y \geqslant y_{0}$, where $y_{0}=y_{0}(n)=$ $x_{0}(n) / a$. A computation shows that, writing $[t]$ for the integral part of $t$,

(10) $f_{n}(t)=2^{n}\left[\frac{t}{2^{n+1}}\right]+\min \left(2^{n}, 1+[t]-2^{n+1}\left[\frac{t}{2^{n+1}}\right]\right)$ for $t \geqslant 0$,

and, of course, $f_{n}(t)=0$ for $t \leqslant 0$. For we may write $t=k 2^{n+1}+s$, where $z$ is a non-negative integer, and $0 \leqslant s<2^{n+1}$. And $\varepsilon_{0} 2^{0}+\varepsilon_{2} 2^{1}+$ $+\ldots+\varepsilon_{n-1} 2^{n-1}$ may be any non-negative integer $p<2^{n}$, while $\varepsilon_{n+1} 2^{n+1}+$ $+\varepsilon_{n+2} 2^{n+2}+\ldots$ may be any number $2^{n+1} q$, where $q$ is any non-negative integer. Thus, we may rewrite (6) as

$$
p+2^{n+1} q \leqslant k 2^{n+1}+s
$$

and $f_{n}(t)=f_{n}\left(k 2^{n+1}+s\right)$ is the number of choices of $p$ and $q$ that make (11) valid. Now for $q=0,1,2, \ldots, k-1$ there are exactly $2^{n}$ choices of $p$ that make (11) hold. So far we have accounted for $k \cdot 2^{n}$ choices. For $q=k+1, k+2, \ldots$, there are no acceptable values of $p$. For $q=k$, if $s \geqslant 2^{n}-1$ then there are $2^{n}$ choices of $p$, while if $s<2^{n}-1$, then there are $[s+1]$ ehoices of $p$. Thus, we have

$$
f_{n}\left(k \cdot 2^{n+1}+s\right)=2^{n} k+\min \left(2^{n},[s+1]\right),
$$

which is equivalent to (10).

Now, writing $y=2^{n+1} k+s$, with $k$ a non-negative integer and $0 \leqslant s<2^{n+1}$ as before, we get

$$
f_{n}(y)=2^{n} k+\min \left(2^{n},[s]+1\right),
$$

(14) $f_{n}\left(y-2^{n} b / a\right)=\max \left\{0,2^{n} k+2^{n}[*]+\min \left(2^{n},\left[s-2^{n} b / a\right]+1-2^{n+1}[*]\right)\right\}$, where

$$
*=\frac{s-2^{n} b / a}{2^{n+1}},
$$

and we remark that [*] $=0$ or -1 according as $s \geqslant 2^{n} b / a$ or $s<2^{n} b / a$, respectively.

$$
\text { We now let }
$$

$$
g(y)=g_{n}(y)=2^{n} k+\min \left(2^{n}, s\right),
$$

(17) $\quad h(y)=h_{n}(y)=\max \left\{0,2^{n} k+2^{n}[*]+\min \left(2^{n}, s-2^{n} b / a-2^{n+1}[*]\right)\right\}$, and let

$$
B_{n}^{\prime}=\frac{1}{a} \sup _{y \geqslant y_{0}(n)} \psi(y)
$$

where

$$
\psi(y)=\psi_{n}(y)=\frac{g_{n}(y)+h_{n}(y)}{y} .
$$

Since $\left|B_{n}^{\prime}-B_{n}\right| \leqslant \frac{2}{y_{0}(n)}$, we see that $B=\limsup _{n \rightarrow \infty} B_{n}^{\prime}$. We now compute $B_{n}^{\prime}$.

Case 1. $s<2^{n} b / a$. Here $[*]=-1, g(y)=2^{n} k+s$, and $h(y)=$ $=\max \left\{0,2^{n} k-2^{n}+\min \left(2^{n}, s-2^{n} b / a+2^{n+1}\right)\right\}$, but $2^{n+1}+s-2^{n} b / a \geqslant$ $2^{n+1}-2^{n} b / a=2^{n}(2-b / a) \geqslant 2^{n}$ since $b / a=\alpha / \beta \leqslant 1$, so that $h(y)=2^{n} k$, and $\sup _{1} \psi(y)=\left(2^{n+1} k+s\right) /\left(2^{n+1} k+s\right)=1$. There are three more cases, in all of which [*] $=0$ since $s \geqslant 2^{n} b / a$.

Case 2. $2^{n} b / a \leqslant s \leqslant 2^{n}$. Here $g(y)=2^{n} k+s$ and $h(y)=2^{n} k+$ $+\min \left(2^{n}, s-2^{n} b / a\right)=2^{n} k+s-2^{n} b / a$ since $s-2^{n} b / a \leqslant 2^{n}$. Hence

$$
\psi(y)=\frac{2^{n+1} k+2 s-2^{n} b / a}{2^{n+1} k+s},
$$

and an elementary computation shows that

$$
\sup _{2} \psi(y)=\frac{g\left(2^{n}\right)+h\left(2^{n}\right)}{2^{n}}=2-\frac{b}{a} .
$$

Case 3. $2^{n} \leqslant s \leqslant 2^{n}+2^{n} b / a$. Here, $g(y)=2^{n} k+2^{n}$ and $h(y)=$ $2^{n} k+\min \left(2^{n}, s-2^{n} b / a\right)=2^{n} k+s-2^{n} b / a$, and hence

$$
\psi(y)=\frac{2^{n+1} k+2^{n}+s-2^{n} b / a}{2^{n+1} k+s}=1+\frac{2^{n}-2^{n} b / a}{2^{n+1} k+s},
$$


and an elementary computation shows that

$$
\sup _{3} \psi(y)=\frac{g\left(2^{n}\right)+h\left(2^{n}\right)}{2^{n}}=2-\frac{b}{a} .
$$

Case 4. $2^{n}+2^{n} b / a<s<2^{n+1}$. Here $g(y)=2^{n} k+2^{n}$ and $h(y)=$ $2^{n} k+\min \left(2^{n}, s-2^{n} b / a\right)=2^{n} k+2^{n}$ so that

$$
\psi(y)=\frac{2^{n+1} k+2^{n+1}}{2^{n+1} k+s}
$$

and an elementary computation shows that

$$
\sup _{4} \psi(y)=\frac{g\left(2^{n}+2^{n} b / a\right)+h\left(2^{n}+2^{n} b / a\right)}{2^{n}+2^{n} b / a}=\frac{2}{1+b / a} .
$$

So we must compare the three numbers $2-b / a, 1,2(1+b / a)^{-1}$. Now each of them is $\geqslant 1$, and $2-b / a \geqslant 2(1+b / a)^{-1}$, as an elementary estimate shows. Hence $B_{n}^{\prime}=(2-b / a) / a=\beta\left(2 \alpha / \beta-\alpha^{2} / \beta^{2}\right)$, and the result follows, on letting $n \rightarrow \infty$.

\section{Bounded error terms.}

THEOREM 2. If there are constants $c_{1}$ and $c_{2}$ so that for all $x>0$ we have

then

and

$$
x-c_{1} \leqslant S(x) \leqslant x+c_{2},
$$

Finally, we have

$$
k_{n} \leqslant 2^{n}+c_{1} \quad \text { for all } n
$$

so that if the $k_{n}$ are integers, then $k_{n}=2^{n}$ for all sufficiently large $n$.

Proof. As before, if $x<k_{n}$, then $S(x) \leqslant 2^{n}$. Thus $k_{n}-c_{1} \leqslant 2^{n}$ and (21) is established. Now let

Then

$$
K_{n}=k_{0}+k_{1}+\ldots+k_{n-1} \text {. }
$$

$$
K_{n}+c_{2} \geqslant S\left(K_{n}\right) \geqslant 2^{n} .
$$

We next prove that

$$
2 k_{n}>K_{n}
$$

for all $n$ satisfying $2^{n-1}-n c_{1}-c_{2}>0$. For suppose that $2 k_{n} \leqslant K_{n}$. Then for each choice of $\varepsilon_{0}, \ldots, \varepsilon_{n-1}$, at least one of the sums

$$
k_{n}+\sum_{j=0}^{n-1} \varepsilon_{j} k_{j} \quad \text { or } \quad k_{n}+\sum_{j=0}^{n-1}\left(1-\varepsilon_{j}\right) k_{j}
$$

is less than $K_{n}$, so that in this case we would have $S\left(K_{n}\right) \geqslant 2^{n}+2^{n-1}$. According to (21) and (23), we would have

$$
2^{n}+n c_{1}+c_{2} \geqslant K_{n}+c_{2} \geqslant S\left(K_{n}\right) \geqslant 2^{n}+2^{n-1},
$$

and the assertion is proved. Under the hypothesis of (22), we have $2 k_{n}>K_{n}$. Now for each $y$ with

we have

$$
0 \leqslant y<2 k_{n}-K_{n}
$$

$$
K_{n}+y+c_{2} \geqslant S\left(K_{n}+y\right) \geqslant 2^{n}+S\left(K_{n}+y-k_{n}\right),
$$

where the second term on the right counts the number of $\varepsilon_{0}, \ldots, \varepsilon_{n-1}$ for which

$$
k_{n}+\sum_{j=0}^{n-1} \varepsilon_{j} k_{j} \leqslant K_{n}+y .
$$

Hence

$$
K_{n}+y+c_{2} \geqslant 2^{n}+K_{n}+y-k_{n}-c_{1},
$$

and $(22)$ is established.

Now we choose $p$ so that $2^{p-1}>c_{1}+c_{2}$. Then

$$
K_{n} \leqslant 2^{n}+K_{p} \quad \text { for all large } n \text {. }
$$

For, assume that $n$ is so large that (22) holds, that $n>p$, and that $k_{n+1}>2^{n}$. Then if (28) fails, there would exist at least $2^{p}$ choices of $\varepsilon_{0}, \varepsilon_{1}, \ldots, \varepsilon_{n-1}$ for which

$$
\sum_{j=0}^{n-1} \varepsilon_{j} k_{j}>2^{n}
$$

namely all choices with $\varepsilon_{p+1}=\ldots=\varepsilon_{n-1}=1$. Since there are at most $S\left(c_{1}+c_{2}\right)$ sums not exceeding $2^{n}$ in which one of the summands is $k_{n}$, and no such sums in which one of the summands is $k_{n+1}$ or larger, we obtain

(29) $2^{n}-c_{1} \leqslant S\left(2^{n}\right) \leqslant 2^{n}-2^{p}+S\left(c_{1}+c_{2}\right) \leqslant 2^{n}-2^{p}+c_{1}+c_{2}+c_{2}$, which is contrary to the hypothesis that $2^{p}>2 c_{1}+2 c_{2}$.

If $\sum\left|k_{n}-2^{n}\right|=\infty$, then according to $(24)$,

$$
\sum_{k_{n}>2^{n}}\left(k_{n}-2^{n}\right)=\infty
$$


Thus, we could choose $n_{1}<n_{2}<\ldots<n_{r}$, with $n_{1}$ so large that (22) and (28) hold for $n \geqslant n_{1}$, with

and such that

$$
K_{n_{1}}>K_{n^{\prime}}+c_{1}+c_{2} \text { and } \quad k_{n_{j}}>2^{n_{j}}
$$

$$
A=\sum_{j=1}^{r} k_{n_{j}}>\sum_{j=1}^{r} 2^{n_{j}}+c_{1}+1=B+1+c_{1} .
$$

We now show that

$$
\sum 2^{m_{j}}>B \text { implies } \sum \sum k_{m_{j}}>A \text {. }
$$

This is obvious if $\left\{n_{j}\right\}$ is a subset of $\left\{m_{j}\right\}$. If not, let $n_{s}$ be the largest $n$ not contained in $\left\{m_{j}\right\}$. It follows that

$$
\begin{aligned}
\sum k_{m_{j}} & \geqslant A+k_{n_{s}+1}-\sum_{j=1}^{s} k_{n_{j}} \geqslant A+k_{n_{s}+1}-K_{n_{s}+1}+K_{n_{1}} \\
& \geqslant A+2^{n_{s}+1}-c_{1}-c_{2}-2^{n_{s}+1}-K_{p}+K_{n_{1}}>A .
\end{aligned}
$$

Hence $S(A)$ is no greater than the number of sums of powers of 2 that do not exceed $B$, and this number is at most $B+1$. Hence $S(A) \leqslant B+$ $+1<A-c_{1}$, contrary to hypothesis.

CoROLLARY. If $-c_{1} \leqslant S(x)-\lambda x \leqslant c_{2}$ for some positive constant $\lambda$ and all $x \geqslant 0$, then

and

$$
\begin{gathered}
k_{n} \leqslant \lambda^{-1} 2^{n}+c_{1} \quad \text { for all } n \\
k_{n} \geqslant \lambda^{-1} 2^{n}-\left(c_{1}+c_{2}\right) \quad \text { if } \quad \lambda^{-1} 2^{n-1}>n c_{1}-c_{2},
\end{gathered}
$$

$$
\sum\left|k_{n}-\lambda^{-1} 2^{n}\right|<\infty .
$$

This result follows by applying Theorem 2 to the sequence $\left\{\lambda k_{n}\right\}$. COROLLARY. If the $k_{n}$ are integers, then the only constants $\lambda$ that can occur above have the form $\lambda=2^{N} / M$, where $N \geqslant 0$ and $M>0$ are integers, and then $k_{n}=\lambda^{-1} 2^{n}$ for all sufficiently large $n$.

The proof is a simple application of (35), and we omit it.

3. Unbounded error terms. The methods of the preceding section can be extended to the case where $S(x)-x$ is unbounded.

THEOREM 3. Suppose that

$$
x-f_{1}(x) \leqslant S(x) \leqslant x+f_{2}(x) \text { for all } x \geqslant 0,
$$

where the $f_{i}$ are continuous, positive, non-decreasing functions, not both

bounded, such that $f_{i}(x) / x \rightarrow 0$ as $x \rightarrow \infty$, and such that $x-f_{1}(x)$ and $x+f_{2}(x)$ are strictly increasing. Let $\varphi_{1}$ and $\varphi_{2}$ be the inverse functions defined by

$$
\begin{gathered}
x=y-f_{1}(y) \Leftarrow y=x+\varphi_{1}(x), \\
x=y+f_{1}(y)+f_{2}(y) \Leftarrow \Rightarrow y=x-\varphi_{2}(x),
\end{gathered}
$$

so that the $\varphi_{i}$ are non-decreasing, $\varphi_{i}(x) / x \rightarrow 0$ as $x \rightarrow \infty$, and $x+\varphi_{1}(x)$, $x-\varphi_{2}(x)$ are strictly increasing for sufficiently large $x$. Then

$$
k_{n} \leqslant 2^{n}+\varphi_{1}\left(2^{n}\right) \text { for all } n,
$$

$$
k_{n} \geqslant 2^{n}-\varphi_{2}\left(2^{n}\right) \text { for all large } n \text {. }
$$

Let $\varphi_{3}$ be the inverse function defined by

\section{Then}

and

where

$$
x=y+f_{2}(y) \Leftarrow \Rightarrow y=x-\varphi_{3}(x) .
$$

$$
K_{n} \geqslant 2^{n}-\varphi_{3}\left(2^{n}\right) \text { for all } n
$$

Finally, if we set

then

$$
\psi(x)=\max \left[f_{1}\left(2^{x+1}\right), \varphi_{2}\left(2^{x}\right)+\varphi_{3}\left(2^{x}\right)+\varphi_{4}\left(2^{x}\right)\right\}
$$

$$
\sum_{0}^{N}\left|k_{n}-2^{n}\right|=O(\psi(N)) .
$$

For example, if $f_{1}(x)$ and $f_{2}(x)$ are both $x^{\alpha}$ for large $x$, then the $\varphi_{i}(x)$ are each asymptotic to a suitable constant multiple of $x^{\alpha}$.

To prove (37), use the inequality $S(x)<2^{n}$ if $x<k_{n}$, as before. To prove (39), use the inequality

$$
K_{n}+f_{2}\left(K_{n}\right) \geqslant S\left(K_{n}\right) \geqslant 2^{n},
$$

also as before. By a method entirely analogous to that of the preceding section, it follows that $2 k_{n}>K_{n}$ for all sufficiently large $n$. And for such $n$, proceeding again as before, we have, for each $y$ with $0 \leqslant y$ $<2 k_{n}-K_{n}$,

$$
\begin{aligned}
K_{n}+y+f_{2}\left(K_{n}+y\right) & \geqslant S\left(K_{n}+y\right) \geqslant 2^{n}+S\left(K_{n}+y-k_{n}\right) \\
& \geqslant 2^{n}+K_{n}+y-k_{n}-f_{1}\left(K_{n}+y-k_{n}\right),
\end{aligned}
$$


so that

$$
k_{n} \geqslant 2^{n}-f_{1}\left(K_{n}+y-k_{n}\right)-f_{2}\left(K_{n}+y\right) \geqslant 2^{n}-f_{1}\left(k_{n}\right)-f_{2}\left(k_{n}\right),
$$

which implies (38).

In order to prove (40), we suppose that

$$
K_{n}>2^{n}+K_{p}
$$

where $n$ is so large that $k_{n+1}>2^{n}$ and $k_{n}>2^{n-1}$. Then, as in the proof of Theorem 2, we get

$$
\begin{aligned}
2^{n}-f_{1}\left(2^{n}\right) & \leqslant S\left(2^{n}\right) \leqslant 2^{n}-2^{p}+S\left(2^{n}-k_{n}\right) \\
& \leqslant 2^{n}-2^{p}+2^{n}-2^{n}+\varphi_{2}\left(2^{n}\right)+f_{2}\left(2^{n}\right)
\end{aligned}
$$

$$
2^{p} \leqslant f_{1}\left(2^{n}\right)+f_{2}\left(2^{n}\right)+\varphi_{2}\left(2^{n}\right)
$$

so that in view of (37), we have

(44) $\quad K_{n} \leqslant 2^{n}+K_{p} \leqslant 2^{n}+2^{p+1} \leqslant 2^{n}+2 f_{1}\left(2^{n}\right)+2 f_{2}\left(2^{n}\right)+2 \varphi_{2}\left(2^{n}\right)$

for all sufficiently large $n$.

Now assume that

$$
\lim \sup \sum_{k=0}^{N}\left|k_{n}-2^{n}\right| / \psi(N)=\infty
$$

In view of (39), this implies that

where

$$
\limsup \sum_{k=0}^{N}\left(k_{n}-2^{n}\right)^{+} / \psi(N)=\infty
$$

$$
\left(k_{n}-2^{n}\right)^{+}=\max \left(0, k_{n}-2^{n}\right) .
$$

We first prove that if $\chi(N)=\log _{2}\left(\varphi_{2}\left(2^{N}\right)+\varphi_{3}\left(2^{N}\right)+\varphi_{4}\left(2^{N}\right)\right)$, then

$$
\sum_{x(N)<n<N}\left(k_{n}-2^{n}\right)^{+} /(\psi(N)+1) \leqslant 1 .
$$

For, let $n_{1}, n_{2}, \ldots, n_{r}$ be the values of $n$ for which

Let

$$
k_{n}>2^{n}, \quad \chi(N)<n_{1}<n_{2}<\ldots<n_{r}<N .
$$

$$
A=\sum_{j=1}^{r} k_{n_{j}}>\sum_{j=1}^{r} 2^{n_{j}}+\psi(N)+1=B+\psi(N)+1 .
$$

Then, as in the proof of Theorem $2, \Sigma 2^{m_{j}}>B$ implies that $\sum k_{m_{j}}>A$.
This is obvious if $\left\{n_{j}\right\}$ is a subset of $\left\{m_{j}\right\}$. If not, let $n_{s}$ be the largest element of $\left\{n_{i}\right\}$ not contained in $\left\{m_{j}\right\}$, so that

$$
\begin{aligned}
\sum k_{m_{j}} & \geqslant A+k_{n_{s}+1}-K_{n_{s}+1}+K_{n_{1}} \\
& \geqslant A+2^{n_{s+1}}-\varphi_{2}\left(2^{n_{s}+1}\right)-2^{n_{s}+1}-\varphi_{4}\left(2^{n_{s}+1}\right)+2^{n_{1}}-\varphi_{3}\left(2^{n_{1}}\right) \\
& >A+2^{x(N)}-\varphi_{2}\left(2^{N}\right)-\varphi_{3}\left(2^{N}\right)-\varphi_{4}\left(2^{N}\right)>A .
\end{aligned}
$$

Hence $S(A)$ is no greater than the number of sums of powers of 2 that do not exceed $B$, so that $S(A) \leqslant B+1$. It follows that

$$
A-f_{1}(A) \leqslant S(A) \leqslant B+1<A-\psi(N),
$$

which leads to a contradiction, since $A \leqslant K_{N}<2^{N+1}$ for all' large $N$. Now we have $1+\varphi(x)<\frac{1}{2} 2^{x}$ for all $x>x_{0}$, and therefore, from $(45)$, if we let

$$
\chi_{0}(N)=N \quad \text { and } \quad \chi_{m+1}(N)=\chi\left(\chi_{m}(N)\right),
$$

then, provided $\chi_{m+1}(N)>x_{0}$, we have

But

$$
\sum_{x_{m+1}(N)<n_{<m}(N)}\left(k_{n}-2^{n}\right)^{+} \leqslant 1+\psi\left(\chi_{m}(N)\right)<\frac{1}{2} 2^{x_{m}(N)} .
$$

$$
2^{x_{m}^{(N)}} \leqslant \psi\left(\chi_{m-1}(N)\right),
$$

so that if $\chi_{m+1}(N)>x_{0}$, we have

$$
\sum_{x_{m+1}(N)<n<x_{m}(N)}\left(k_{n}-2^{n}\right)^{+} \leqslant \frac{1}{2^{m}} \psi(N) .
$$

On adding the inequalities (46) for all suitable $m$, we get

$$
\sum_{n=0}^{N}\left(k_{n}-2^{n}\right)^{+} \leqslant 2 \psi(N)+O(1),
$$

which proves (41) by contradiction.

4. Irregularity of $S(x)$. We say that a function $f$ is slowly oscillating to mean that for each positive constant $a, f(a x) / f(x) \rightarrow 1$ as $x \rightarrow \infty$.

THEOREM 4. It is impossible to have $S(x) \sim x^{\alpha} f(x)$, where $0<\alpha<1$, and $f(x)$ is a continuous positive slowly oscillating function such that $x^{\alpha} f(x)$ is strictly increasing.

Proof. Define the inverse function $g$ by

$$
y=x^{\alpha} f(x) \Leftrightarrow x=y^{1 / \alpha} g(y) .
$$


Then $g$ is also a continuous positive slowly oscillating function. From $S\left(k_{n}-\right) \leqslant 2^{n}$, we get

$$
k_{n}<(1+\varepsilon) 2^{n j a} g\left(2^{n}\right)
$$

for any $\varepsilon>0$ and all sufficiently large $n$, so that

$$
K_{n}=\sum_{m=0}^{n-1} k_{m}<(1+\varepsilon) \sum_{m=0}^{n-1} 2^{m / \alpha} g\left(2^{m}\right) .
$$

On the other hand, we have $S\left(K_{n}\right) \geqslant 2^{n}$, so that

and hence

$$
K_{n}>(1-\varepsilon) 2^{m / \alpha} g\left(2^{n}\right),
$$

$$
\frac{1-\varepsilon}{1+\varepsilon}<\sum_{m=0}^{n-1} 2^{(m-n) / a} \frac{g\left(2^{m}\right)}{g\left(2^{n}\right)}
$$

for all sufficiently large $n$. We show now that (47) is impossible for small $\varepsilon$. We use the result [2] that there is a function $h(x)$ with $h(x) \sim c g(x)$ as $x \rightarrow \infty$, where $c$ is a positive constant, such that $h(x)$ has the representation

$$
h(x)=\exp \int_{1}^{x} \beta(t) t^{-1} d t
$$

where

$$
\beta(t)=o(1) \quad \text { as } \quad t \rightarrow \infty .
$$

It follows from (49) that $h(x) \geqslant x^{-\delta}$ for any $\delta>0$, for all sufficiently large $x$, and the same inequality consequently holds for $g$. It follows that the values of $g(x)$ when $x$ is small do not affect the inequality (47) for large $n$, and that to contradict (47), it is enough to contradict the corresponding inequality for $h$, which by (48) may be written as

$$
\frac{1-\varepsilon}{1+\varepsilon}<\sum_{m=0}^{n-1} \exp \left(-\int_{2^{m}}^{2^{n}}\left\{\frac{1}{\alpha}+\beta(t)\right\} \frac{d t}{t}\right) .
$$

If we now choose $\gamma$ so that $1<\gamma<1 / \alpha$, then for all sufficiently large $t$,

$$
1 / \alpha+\beta(t)>\gamma,
$$

and by the above remarks, there is no loss in assuming this for all $t$. We then have

$$
\frac{1-\varepsilon}{1+\varepsilon}<\sum_{m=0}^{n-1} 2^{\gamma(m-n)}=\sum_{r=1}^{n} 2^{-\gamma r}<\frac{1}{2^{\gamma}-1}
$$

which is a contradiction if $\varepsilon$ is small enough, and the theorem is proved.
On the other hand, it is possible, for each positive integer $\alpha$, to have $S(x) \sim c x^{\alpha}$, where $c$ is any positive constant. For example, if we let $K$ consist of $\alpha$ copies of $\left\{2^{n}\right\}, n=0,1, \ldots$, then a simple computation shows that $S(x) \sim c_{a} x^{\alpha}$. Perhaps, then, it is impossible to have $S(x) \sim f(x) x^{\alpha}$, where $\alpha>0, f(x)$ is slowly oscillating, and $x^{\alpha} f(x)$ is strictly increasing, unless $\alpha$ is an integer. We outline here a proof of a partial result in this direction, namely that if $1<\alpha<\alpha_{0}$, for a certain $\alpha_{0}$ (with $1<\alpha_{0}<2$ ) then $S(x) \sim f(x) x^{\alpha}$ is impossible. We treat the case $f(x)=1$ for all $x$; the general case is similar.

In this case, we apply arguments like those above to get $(2-\delta) k_{n}>K_{n}$ for some $\delta>0$ and infinitely many $n$. But then we have

$$
S\left(\left(\frac{1}{2}+\varepsilon\right) K_{n}\right)=2^{n}-S\left(\left(\frac{1}{2}-\varepsilon\right) K_{n}\right),
$$

provided only that $\varepsilon$ is chosen so small that

since for every sum

$$
k_{n}>\left(\frac{1}{2}+\varepsilon\right) K_{n}
$$

$$
\sum_{i=0}^{n-1} \varepsilon_{i} k_{i}<\left(\frac{1}{2}-\varepsilon\right) K_{n}
$$

we have

$$
\sum_{i=0}^{n-1}\left(1-\varepsilon_{i}\right) k_{i}>\left(\frac{1}{2}+\varepsilon\right) K_{n}
$$

But the asymptotic relation

$$
\left(\frac{1}{2}+\varepsilon\right)^{\alpha} K_{n}^{a} \sim 2^{n}-\left(\frac{1}{2}-\varepsilon\right)^{\alpha} K_{n}^{\alpha}
$$

cannot hold identically in $\varepsilon$ unless $\alpha=1$, which is excluded, and the result is proved.

\section{References}

[1] Basil Gordon and L. A. Rubel, On the density of sets of integers possessing additive bases, Illinois J. Math. 4 (1960), pp. 367-369.

[2] J. Korevaar, T. van Aardenne-Ehrenfest and N. G. de Bruijn, A note on slowly oscillating functions, Nieuw. Arch. Wisk. (2), 23 (1949), pp. 77-86.

\section{Reçu par la Rédaction le 29. 9. 1963}

